\title{
EVALUATION OF LOWER URINARY TRACT SYMPTOMS OF OUTPATIENTS WITH PROSTATIC DISEASE : THE RELATIONSHIP AMONG UFM, TRUS, AND IPSS AND QOL SCORE
}

\author{
Kazuhiro Suzuki ${ }^{1,2)}$, Yoshihiro Totsuka ${ }^{1)}$, Kazuhisa Matsumoto ${ }^{1,2)}$, \\ Yoshihiro Ono ${ }^{1)}$, Hiroaki Tsuji ${ }^{2)}$, Kohei Kurokawa ${ }^{1)}$, \\ Takanori Suzuki ${ }^{1)}$ and Hidetoshi Yamanaka ${ }^{1)}$ \\ Department of Urology, Gunma University School of Medicine ${ }^{1)}$ \\ and Division of Urology, Honjo General Hospital ${ }^{2}$
}

\begin{abstract}
To assess the relationship between urinary tract symptoms and objective parameters in patients with prostatic disease, international prostatic symptom score (IPSS), quality of life score (QOL score), and parameters of transrectal ultrasonography (TRUS) and uroflowmetry (UFM). In new patients, Qmax in UFM was correlated with IPSS and QOL score. Parameters of TRUS were not correlated with symptoms. Total score of voiding component of IPSS was more highly correlated with QOL score than that of filling component of IPSS. In treated patients, patients with severe QOL score had low Qmax, high total score IPSS, and high scores of individual questions of IPSS. In patients with prostate cancer, irritative symptoms got severe and Qmax was not correlated with symptoms. To evaluate lower urinary tract symptoms in reference to other objective parameters is thought to be of great use in managing patients with prostate disease.
\end{abstract}

Key words : IPSS, QOL score, UFM, TRUS, Prostatic disease (Kitakanto Med.J. 47 (5) : 305 309, 1997)

\section{INTRODUCTION}

The number of patients with lower urinary tract symptoms has been increased ${ }^{1}$. The standardized measurement of such symptoms has become a frequently used tool ${ }^{2}$. International prostatic symptom score (IPSS) and quality of life score (QOL score) were widely adopted ${ }^{3)}$. Most studies on evaluation of relationship between lower urinary tract symptoms and other subjective parameters has been reported from large urological faculties ${ }^{4 \sim 8)}$. We report in this paper the observation of lower urinary tract symptoms of outpatients with prostatic disease visiting Division of Urology, Honjo General Hospital. Honjo General Hospital is located at the north part of Saitama prefecture and is a medium size hospital. Division of Urology is a related urological unit of Department of
Urology, Gunma University School of Medicine and ordinary urological practice is performed.

\section{PATIENTS AND METHODS}

Total 130 outpatients with prostatic disease were enrolled in this study during July, 1996 and March, 1997. Ages of patients ranged from 48 to 88 years old with a mean age of 69.1 . Of 130 patients, 36 were new patients with a mean age of 68.4 years old and none of them had sign of prostate cancer on digital rectal examination (DRE), transrectal ultrasonography (TRUS) and serum prostatic specific antigen (PSA) level. Twenty-two patients had histologically proven prostate cancer. Their ages ranged from 63 to 88 years old with a mean age of 74.0. They were terated with hormone therapy for 1 to 120 mo. Retropubic radical prostatectomy was performed on 1 patient. Other 72

Received : June 10, 1997

Address : KAZUHIRO SUZUKI, MD

Department of Urology, Gunma University School of Medicine, 3-39-22 Showa-machi, Maebashi 371, JAPAN. 
patients of 130 had benign prostate hypertrophy (BPH) clinically. Six of them had chronic prostatitis and other 6 had neurogenic bladder. They were treated with medication. Alpha-1 blocker was administered to 50 patients with combination of pollen exudate derivative, antiandrogen to 9, pollen exudate derivative to 5 , herbal medicine to 1 , cholinergic agents to 7 , respectively. Transurethral resection of prostate and endourethrotomy were performed on 6 and 1 patient, respectively. Distribution of treatment duration was shown in table 4.

IPSS consists of seven question as shown in table 1 . Total score of IPSS questions 2, 4, and 7 represents the filling component of the IPSS, while that of question 1 , 3 , 5, and 6 represents the voiding component. The patients has to indicate the presence of these symptoms on a scale varying from zero (not at all) to 5 (almost always). The scores on the individual questions are summed to give a total index (range O-35). QOL score consists of 7 scales as shown in table 1.

Uroflowmetry (UFM) was performed using

Table 1 Questionnaire of IPSS and QOL score

Questionnaire of IPSS

1. How often have you had a sensation of not emptying your bladder completely after you finished urination?

2. How often have you had to urinate again less than 2 hours after you finished urination?

3. How often have you found you stopped and started again several times when you urinated?

4. How often have you found it difficult to postpone urination?

5. How often have you had a weak stream?

6. How often have you had to push or strain to begin urination?

7. How many times did you most typically get up to urinate from the time you went to bed at night until the time you got up in the morning?

Questionnaire and scales of QOL score

If you were to spend the rest of your life with your urinary condition just the way it is now, how would you feel about that?

Scales : $0:$ delighted, $1:$ pleased, $2:$ mostly satisfied, 3 : mixed, about equally satisfied and dissatisfied, $4:$ mostly dissatisfied, 5: unhappy, 6: terrible

URODYN 1000 (Dantek, Denmark). Maximum flow rate (Qmax) and mean flow rate (Qmean) were measured.

Transrectal ultrasonography (TRUS) was performed using SSA-240A (Toshiba Med, Tokyo) with a biplane probe, PVL-51S. Whole prostate volume and transition zone volume (TZ) were estimated. $\mathrm{TZ}$ index was calculated as rate of volume of $\mathrm{TZ}$ to that of whole prostate.

\section{RESULTS}

\section{Aspects of new patients}

UFM and TRUS were performed on 31 and 33 patients, respectively. Qmax ranged from 3.6 to $22.4 \mathrm{ml} / \mathrm{s}$ with a mean of $10.3 \mathrm{ml} / \mathrm{s}$, and Qmean ranged from 1.6 to $16.2 \mathrm{ml} / \mathrm{s}$ with a mean of $5.5 \mathrm{ml} / \mathrm{s}$. Whole prostatic volume ranged from 13.5 to $107.6 \mathrm{cc}$, and $\mathrm{TZ}$ volume ranged from 1.61 to $53.8 \mathrm{cc}$. TZ index ranged from 0.092 to 0.73 . IPSS and QOL score were obtained from 36 patients. Total score of IPSS ranged from 1 to 28 with a mean of 13.1. Mild symptoms, score 0-7, were found in 10 , moderate symptoms, scores $10-19$, in 21 , and severe symptoms, score $20-35$, in 5 , respectively. QOL scores ranged from 1 to 6 with a mean of 4.2 . QOL score was correlated with total score of IPSS $(r=$ 0.68). Total score of voiding component of IPSS was more highly correlated with QOL score than that of filling component of IPSS ( $r=0.65$ vs $r=0.47$ ). Relationship between score of individual questions and total score of IPSS or QOL score is shown in table 2.

Relationship among parameters were shown in table 3. Qmax and Qmean were correlated with total score of IPSS $(r=0.61$ and $r=0.61)$. Total score of filling component of IPSS were more highly correlated with Qmax than that of voiding component of IPSS $(r=-0.58$ vs $r=-0.44)$. However, prostate volume both of whole and $\mathrm{TZ}$ or $\mathrm{TZ}$ index was not correlated with total score of IPSS or QOL score. Qmax was not correlated with prostate volume of whole and $\mathrm{TZ}$ or $\mathrm{TZ}$ index $(\mathrm{r}=-0.25,-0.33$, and -0.37 , respectively).

Table 2 Relationship between individual score and IPSS total score or QOL score in new patients

\begin{tabular}{lcc}
\hline IPSS questions & IPSS total score & QOL score \\
\hline 1. Bladder emptying & 0.68 & 0.45 \\
2. Daytime frequency & 0.61 & 0.30 \\
3. Intermittency & 0.67 & 0.39 \\
4. Urgency & 0.76 & 0.52 \\
5. Stream & 0.63 & 0.65 \\
6. Straining & 0.44 & 0.33 \\
7. Nocturia & 0.61 & 0.33 \\
\hline
\end{tabular}

Values are expressed as correlation coefficient.

Table 3 Relationship between UFM or TRUS parameters and IPSS total or QOL score in new patients

\begin{tabular}{lcc}
\hline & IPSS total score & QOL score \\
\hline UFM & & \\
Qmax & -0.61 & -0.45 \\
Qmean & -0.61 & -0.43 \\
TRUS & & \\
whole vol & 0.08 & -0.12 \\
TZ vol & 0.20 & -0.005 \\
TZ index & 0.23 & 0.11 \\
\hline
\end{tabular}

Values are express as correlation coefficient. 
Symptoms and objective parameters in prostatic disease

Table 4 Patient characteristics of treated patients with benign prostatic disease

\begin{tabular}{|c|c|c|c|c|c|c|c|}
\hline \multirow{2}{*}{$\begin{array}{l}\text { Treatment } \\
\text { duration } \\
\text { (mo) }\end{array}$} & \multirow{2}{*}{$\mathrm{n}$} & \multirow{2}{*}{$\begin{array}{l}\mathrm{Qmax} \\
(\mathrm{ml} / \mathrm{s})\end{array}$} & \multicolumn{2}{|c|}{ Prostate vol (cc) } & \multirow{2}{*}{$\mathrm{TZ}$ index } & \multirow{2}{*}{$\begin{array}{l}\text { IPSS } \\
\text { total } \\
\text { score }\end{array}$} & \multirow{2}{*}{$\begin{array}{l}\text { QOL } \\
\text { score }\end{array}$} \\
\hline & & & whole & $\mathrm{TZ}$ & & & \\
\hline$<1$ & 17 & 11.0 & 25.1 & 6.7 & 0.21 & 14.5 & 4.4 \\
\hline $1-3$ & 13 & 11.5 & 41.5 & 12.0 & 0.22 & 7.3 & 1.9 \\
\hline $3-6$ & 8 & 10.7 & 28.0 & 9.6 & 0.34 & 9.5 & 2.8 \\
\hline $6-12$ & 7 & 8.8 & 36.3 & 4.5 & 0.10 & 14.9 & 3.9 \\
\hline $12-24$ & 14 & 11.2 & 27.4 & 7.4 & 0.27 & 11.1 & 3.1 \\
\hline$<24$ & 13 & 14.2 & 26.4 & 6.5 & 0.22 & 11.5 & 2.9 \\
\hline
\end{tabular}

Table 5 Relationship between individual score and QOL score in treated patients with benign prostate disease

\begin{tabular}{lcccccc}
\hline \multirow{2}{*}{ IPSS questions } & \multicolumn{5}{c}{ Treatment duration (mo) } \\
\cline { 2 - 6 } & $<1$ & $1-3$ & $3-6$ & $6-12$ & $12-24$ & $<24$ \\
\hline 1. Bladder emptying & - & - & - & - & $\#$ & - \\
2. Daytaime frequency & - & - & - & $\#$ & - & - \\
3. Intermittency & - & - & - & - & - & - \\
4. Urgency & - & - & - & $\#$ & - & - \\
5. Stream & - & $\#$ & - & $\#$ & - & - \\
6. Straining & - & - & $\#$ & $\#$ & - & - \\
7. Nocturia & - & - & - & $\#$ & - & - \\
\hline
\end{tabular}

Table 6 Differences of mean values in severe QOL score counting patients with benign prostate disease

\begin{tabular}{lccc}
\hline & \multicolumn{3}{c}{ Treatment duration (mo) } \\
\cline { 2 - 4 } & $\begin{array}{c}\text { First } \\
\text { visit }\end{array}$ & $\leqq 1 \mathrm{mo}$ & $1 \mathrm{mo}<$ \\
\hline $\mathrm{n}(/$ total $)$ & $20 / 36$ & $10 / 17$ & $10 / 55$ \\
QOL score & 1.4 & 1.0 & 2.5 \\
IPSS total score & 3.8 & 3.3 & 7.2 \\
Qmax & 1.6 & 0.5 & 4.2 \\
IPSS score & & & \\
1. Bladder emptying & - & - & $\#$ \\
2. Daytaime frequency & - & - & $*$ \\
3. Intermittency & - & - & $*$ \\
4. Urgency & $*$ & - & $*$ \\
5. Stream & $*$ & - & $\#$ \\
6. Straining & - & - & $*$ \\
7. Nocturia & - & - & $\#$ \\
\hline
\end{tabular}

\#: difference of mean value $>1.0$

$*$ : difference of mean value $>0.7$

Values are expressed as difference of mean values in patients with 5 or 6 of QOL score vs total patients.

\section{Aspects of treated patients with benign prostatic disease.}

Patient characteristics are shown in table 4. Correlation coefficient between total score of IPSS and QOL score of all patients was 0.66. Table 5 shows the correlation between QOL score and score of individual questions. In high QOL score group of treatment period 6-12 mo, correlation coefficients got high.

\section{Aspects of patient with high QOL score in benign prostatic disease.}

In clinical practice, to relieve the symptoms of patients whose QOL score gets high is an important task. To assess the clinical characteristics of patients with QOL score 5 and 6, differences of total score and QOL score, Qmax, and scores of individual questions were calculated as shown in table 6 .

\section{Aspects of patients with prostate cancer.}

UFM and TRUS were performed on 22 and 19 patients, respectively. The mean values of $\mathrm{Qmax}$ and Qmean were 11.3 and $6.4 \mathrm{ml} / \mathrm{s}$. The mean whole prostate volume was $20.2 \mathrm{cc}$. The mean total score of IPSS and QOL score of 22 patients were 9.2 and 3.1, respectively.

Qmax was not correlated with total score of IPSS $(r=0.06)$, QOL score $(r=0.19)$, whole prostate volume $(\mathrm{r}=0.10)$, and treatment duration $(\mathrm{r}=0.24)$. QOL score was correlated with total score of IPSS $(r=0.70)$, and total score of filling component of IPSS was more highly correlated than that of voiding component as shown in table 7 .

Table 7 Relationship between individual score, and IPSS total score and QOL score in patients with prostate cancer

\begin{tabular}{lcc}
\hline IPSS questions & IPSS total score & QOL score \\
\hline 1. Bladder emptying & $\#$ & - \\
2. Daytaime frequency & - & - \\
3. Intermittency & - & - \\
4. Urgency & $\#$ & - \\
5. Stream & - & - \\
6. Straining & - & - \\
7. Nocturia & $\#$ & \\
\hline
\end{tabular}




\section{DISCUSSION}

BPH causes prostatic enlargement, which in turn may lead to bladder outlet obstruction and lower urinary tract symptoms. Clinical BPH has been characterized by the combination of three parameters: lower urinary tract symptoms, bladder outlet obstruction and increased prostate volume due to hyperplasia $^{9}$. We investigated in this study the relationship between such symptoms and other parameters. To evaluate symptom severity, IPSS is now thought to be valid $^{2 \sim 5)}$ and is accepted in spite of cultural variation $^{10)}$. Treatment policy for patients with lower urinary tract symptoms is to relieve the symptoms. Therefore the investigation of change of IPSS and QOL score is an important aspect.

In new patients, Qmax and Qmean were correlated with total score of IPSS. Din et al. reported the relationship between IPSS and urodynamic study parameters with pressure-flow study ${ }^{7}$. The parameters were weakly correlated with IPSS. Other report of the same authors demonstrated the similar results of our study ${ }^{8}$. IPSS and results of UFM were significantly but weakly correlated. No correlation between IPSS and results of prostate volume measurements was reported by several authors ${ }^{8,11,12)}$, and our result supported it. The analysis of scores of individual questions of IPSS was meaningful to focus on the chief complaint. In our study, the questions about urgency (No.4) and stream (No.5) were highly correlated with both total score of IPSS and QOL score. In clinical practice, to relieve severe symptoms is an important task. We investigated the characteristics of patients with severe QOL score. In new patients, the scores on urgency and stream got high, although difference of the average of Qmax is only $1.6 \mathrm{ml} / \mathrm{s}$. In treated patients, differences of total score of IPSS, Qmax, and individual scores of IPSS were rather high. These patients did not responded to medication, and differences of parameters between in responded patients and in non-responded patients would be increased.

Urinary condition in patients with prostate cancer has not been frequently documented, for the response of cancer to therapy is of great attention. However, the importance of QOL in prostate cancer patients has been recently reported ${ }^{13,14}$. In our study, the filling component of IPSS were correlated with severity of symptoms. The voiding component such as, intermittency, stream, or straining, was not so highly correlated with not so highly correlated with severity. Silva et $\mathrm{al}^{15)}$ stated that aspects of performance status, symptoms from prostate, pain, general fatigue, psychological condition, sexual activities, and social activities were important as QOL in patients with prostate cancer. In our study, only a part of symptoms from prostate was investigated. To evaluate QOL in prostate cancer, other aspects should be investigated.

In summary, Qmax in UFM was correlated with urinary tract symptoms in new patients. Prostate volume has no correlation with Qmax or symptoms. In treated patients, patients with severe QOL score had low Qmax, high total score IPSS, and high scores of individual questions of IPSS. In patients with prostate cancer, irritative symptoms got severe. To evaluate lower urinary tract symptoms in reference to other objective parameters is thought to be of great use in managing patients with prostate disease.

\section{REFERENCES}

1) Oestering JE. Benign prostatic hyperplasia. Medical and minimally invasive treatment options. N Engl J Med 1995; 332 : 99-109.

2) Riehmann R, Jesper B, Polishuk PV, et al. Symptom scores in benign prostatic hyperplasia. Urology $1997 ; 49$ : 10-18.

3) Barry MJ, Fowler FJ, O'Leary MP, et al. The American Urological Association symptom index for benign prostatic hyperplasia. J Urol 1992; 148 : 1549-1557.

4) Barry MJ, Cockett ATK, Holtgrewe HL, et al. Relationship of symptoms of prostatism to commonly used physiological and anatomical measures of the severity of benign prostatic hyperplasia. J Urol 1993; 150 : 351-358.

5) Reynard J, and Abrams P. Symptoms and symptoms scores in BPT. Scand J Urol Nephrol 1994 ; 157: 137-145.

6) Ko DSC, Fenster HN, Chambers K, et al. The correlation of multichannel urodynamic pressureflow studies and American Urological Association symptom index in the evaluation of benign prostatic hyperplasia. J Urol 1995 ; 154 : 396-398.

7) Din KEE, Kiemeney LALM, Wildt MJAM, et al. The correlation between bladder outlet obstruction and lomer urinary tract symptoms as measured by the international prostate symptom score. J Urol 1996; 156 : 1020-1025.

8) Din KEE, Kiemeney LALM, Wildt MJAM, et al. Correlation between uroflowmetry, prostate volume, postvoid residue, and lower urinary tract symptoms as measured by the international prostate symptom score. Urology $1996 ; 48: 393-397$.

9) Hald T. Urodynamics in benign prostatic hyperplasia: A survey. Prostate, suppl. 1989;2: 69-77.

10) Netto NR, Lima ML, Andrade EFM, et al. Latin 
american study on patient acceptance of the international prostate symptom score (IPSS) in the evaluation of symptomatic benign prostatic hyperplasia. Urology 1997 ; 49 : 4-49.

11) Chapple CR. Correlation of symptomatology, urodynamics, morphology and size of the prostate in benign prostatic hyperplasia. Curr Opinion Urol 1993 ; 3 : 5-9.

12) Simpson RJ, Fisher W, Lee AJ, et al. Benign prostatic hyperplasia in an unselected communitybased population: A survey of urinary symptoms, bothersomeness and prostatic enlargement. Brit $\mathbf{J}$
Urol 1996; 77 : 186-191.

13) Kurokawa K, Suzuki T, Suzuki K, et al. Quality of life after radical prostatectomy. Kitakanto Med J 1996 ; 46 : 301-307.

14) Matchar DB, McCrory DC, and Bennett CL. Treatment considerations for persons with metastatic prostate cancer: Survival versus out-ofpocket costs. Urology 1997 ; 49 : 218-224.

15) Silva $C$ and Aaronson N. Quality of life assessment in prostatic cancer. Prog Clin Biol Res. $1988 ; 260$ : 119-121. 